\title{
On the modeling of electrical boundary layer (electrode layer) and derivation of atmospheric electrical profiles, eddy diffusion coefficient and scales of electrode layer
}

\author{
MADHURI N KULKARNI \\ Indian Institute of Tropical Meteorology, Dr. Homi Bhaba Road, Pune 411 008, India. \\ e-mail:mnkulk2005@gmail.com
}

\begin{abstract}
Electrode layer or electrical boundary layer is one of the charge generators in the global atmospheric electric circuit. In spite of this we find very few model studies and few measurements of it in the literature. Using a new technique it is shown that in this layer, the space charge density varies exponentially in vertical. A new experimental method based on the surface measurements is discussed to determine all the characteristic scales and an average electrical and meteorological state of an electrode layer. The results obtained are in good agreement with the previous studies. So, it is suggested that an exponential space charge density profile will no longer be an assumption in the case of electrode layer studies. The profiles of atmospheric electric field and electrical conductivity are also derived and a new term named as electrode layer constant is introduced.
\end{abstract}

\section{Introduction}

The electrical structure of electrode layer depends on the different charge generating processes occurring near the surface of the earth and then successive turbulent mixing of the generated charge particles in an atmospheric environment (e.g., bursting of bubbles on the ocean surface, ionization due to surface radioactivity over land, injection of pollutants into the atmosphere, etc.). Vertical profiles of atmospheric electrical parameters in the surface layer are characterized by these processes. So it is necessary to study these environmental changes, their turbulent transfer and corresponding changes in the electrical state of electrode layer or electrical boundary layer to draw conclusions regarding changes in atmospheric electrical parameters with respect to environmental conditions. Atmospheric eddy diffusion coefficient is the main parameter which is a measure of this turbulent transport and characterizes vertical profiles of atmospheric electrical parameters in the surface layer (e.g., Hoppel and Gathman 1971; Kulkarni and Kamra 2001).

As mentioned above, electrode layer acts as one of the charge generators in the global atmospheric electric circuit. The main purpose of the present study is to find easier way to study the electrical state of electrode layer with respect to environmental conditions. It can be studied by determining its characteristic scales. These scales are: atmospheric eddy diffusion coefficient, steady state atmospheric electric field, steady state atmospheric electrical conductivity and thickness of electrode layer (Willett 1978). A new simple method which serves this purpose is suggested here.

Some attempts were made to determine the eddy diffusion coefficient by atmospheric electrical method. There are basically three approaches to determine an exchange or eddy diffusion coefficient from the atmospheric electrical measurements,

(1) measurement of atmospheric electrical parameters aloft: in this case conductivity or

Keywords. Atmospheric electrical method; electrode layer; Poisson's equation; surface measurements. 
ion number density profiles are obtained from which profile of aerosol concentration is derived. These profiles are further used for calculating eddy diffusion coefficient.

(2) Making measurements of radioactivity aloft: the distribution of radioactive gases and their daughter products depends upon their half lives as well as mixing intensity of the atmosphere. If airmass travels longer distance with intense vertical mixing, the quantity of radon it carries will be more. On a mountain peak at $3 \mathrm{~km}$ a.s.l. above an inversion, the radon concentration observed was almost $0.1 \%$ of that measured in surrounding valley. So, in this approach the measurements of radioactive gases are made at three mountain stations located closely with each other observing continuously the mean exchange intensity in the layer between these (Dolezalek 1978).

(3) Making near surface measurement of atmospheric electric field and space charge density which requires some of the model generated results for different aerosol concentrations and different surface electric fields as a base frame for comparison (Hoppel and Gathman 1971). A brief discussion of the above three is given by Dolezalek (1978). These three approaches are not simple and it becomes difficult to use them often and in frequently varied environmental conditions. So, there is a need to develop a suitable and easier method to determine the eddy diffusion coefficient from atmospheric electrical measurements.

Kawano (1957) has presented some analytical treatment of equations from the atmospheric electricity to estimate the eddy diffusion coefficient with some assumptions and estimated the diurnal variation of the coefficient of eddy diffusivity. In all his calculations he had assumed the eddy diffusivity to be independent of height.

As mentioned above, Hoppel and Gathman (1971) have developed model for open ocean to determine eddy diffusion coefficient under the following conditions:

- These are open ocean measurements so they are away from the surface radioactivity disturbances. Therefore, they have not considered surface radioactivity in their model equations.

- Aitken nuclei count, surface electric field and space charge density at $1 \mathrm{~m}$ should be known. They incorporated single fixed value of ionization due to cosmic rays in their equations. In their solutions, the space charge at $1 \mathrm{~m}$ is a function of existing nuclei concentration, surface electric field and mixing intensity of the atmosphere. So, by measuring surface electric field and space charge density at $1 \mathrm{~m}$ and comparing these measurements with numerical solutions, one can determine eddy diffusion coefficient.

- There should not be any additional source of positive charge, i.e., there should not be nearby industrial smoke or high tension lines to create charge packets which will disturb electrode effect space charge.

- The conditions should be horizontally homogeneous and the natural electrode layer should not be disturbed by any obstacle, i.e., tall structures like hill or multi-storied building.

Since the atmospheric electrical parameters respond to various environmental parameters, it becomes difficult to study them in isolation. To overcome this difficulty to some extent, one of the ways is to do the scale analysis of model equations by the characteristic scales of electrode layer. This procedure is suggested by Willett (1978). In order to non-dimensionalize the parameters in the model, he has explained the scale analysis procedure appropriate for the electrode layer studies.

Markson and Sedlacek (1981) give the advantage of the atmospheric electric measurements for studying boundary layer fluxes especially studying turbulent flux of space charge in boundary layer. This is easy to measure due to its passive nature. Further, they point out that for atmospheric electrical measurements, one can get excellent signal-to-noise ratio.

Hoppel (1967), Hoppel and Gathman (1971), Willett $(1978,1983)$ show that the solutions of the models for electrode layer are asymptotic. When the steady state conditions are reached, these asymptotic values are attained by different electrical parameters. As mentioned before, these steady state asymptotic electric field and conductivity, thickness of electrode layer and atmospheric eddy diffusion coefficient are the characteristic scales of electrode layer.

Atmospheric conditions are quite variable from time to time and all the above models are based on the specific boundary conditions. Therefore, it becomes difficult to use them on routine basis. So, it is thought to find some experimental method which will involve less expensive and less complicated instrumentation and computer power for studying electrode layer. Moreover, in the literature on atmospheric electricity we find studies on the basic parameters of atmospheric electricity in connection with different meteorological parameters but not on the characteristic scales of electrode layer and the corresponding meteorological parameters. In this paper, we discuss a new method to estimate eddy diffusion coefficient and other 
characteristic scales of electrode layer. The method described here makes use of direct surface measurements of atmospheric electrical parameters and does not require any numerical model solutions for comparison which have restrictions of certain boundary conditions. These actual measurements are to be used for deriving characteristic parameters or scales of the electrode layer in a simple way which are earlier derived only by numerical models under certain conditions. While implementing this method, the basic physics of electrode layer remains the same as it is in the previous theoretical models. In the first part of the paper, equations and methodology are discussed and in the second, we discuss results.

\section{Methodology}

In the atmospheric electricity, the earth's surface is one electrode and electrode layer or electrical boundary layer is a region near the surface of the earth in which profiles of atmospheric electrical parameters are affected by the surface effects (e.g., surface radioactivity) and turbulence. We use the following assumptions which are quite common in atmospheric electricity:

\section{Assumptions:}

- horizontally homogeneous and steady state conditions are assumed and Poisson's equation is considered in only one dimension, i.e., in vertical,

- to use the given profile of eddy diffusion coefficient in surface layer,

- total current $=$ conduction current + convection current. Here we neglect transport due to molecular diffusion as it is only important in the vicinity of the earth's surface, and

- we use the equations appropriate for electrode layer where the strength of atmospheric electric field decreases as we go away from the surface.

Many researchers have previously used, observed or given with some assumptions, exponential profile of space charge density, e.g., Clark (1958); Kraakevik (1958); Varshneya (1958); Israel (1973); Raina (1984); Israelsson et al (1993) and Tammet et al (1996). Here profile of space charge density is derived from the steady state current density equation in turbulent conditions. Transport by molecular diffusion is neglected as it is negligible as compared to turbulent diffusion. Further, it is shown that the derived profile of space charge density is exponential in nature. A region very near to the surface of the earth is excluded where molecular diffusion is important. Since this type of mathematical treatment is given for the first time, all steps in the derivation are given below though it involves simple mathematics.
The atmospheric electrode layer is a region of space charge formed near the earth's surface in which strength of electric field decreases as we go away from the surface. Also, from the physics of electrode layer, in this region we have decreasing positive space charge density as we go away from the surface. As mentioned above in the assumptions, under the steady state conditions in electrode layer we have nonzero space charge density $(\rho)$ and its gradient $(d \rho / d z)$. Some mathematical arguments supporting this can be given as follows.

\subsection{To prove that space charge density varies with altitude in electrode layer,

$$
\text { i.e., } d \rho / d z \neq 0
$$

The steady state fair weather current density $I_{t}$ in a horizontally homogeneous atmosphere can be written as:

$$
I_{t}=E(z) \lambda(z)-K(z) \frac{d \rho}{d z}
$$

where $E(z)$ is the atmospheric electric field, $\lambda(z)$ is the total conductivity and $K(z)$ is the eddy diffusion coefficient at altitude $z . E(z) \lambda(z)$ is the conduction current density and $(K(z) d \rho / d z)$ is the eddy diffusive current due to space charge in vertical at altitude $z$. Eddy diffusion current due to space charge is in the direction opposite to that of its gradient. Also, in fair weather conditions, eddy diffusion current is in the direction opposite to that of conduction current, hence the minus sign before it in equation (1).

In the beginning we prove that $d \rho / d z$ is nonzero in electrode layer and the quantity $[\rho /(d \rho / d z)]$ is well defined.

From the definition of electrode layer given above, it is obvious that in this region $\rho$ always exists, i.e., $\rho \neq 0$. It can be viewed as vertical array of small volumes containing some positive space charge density in it. This space charge density decreases gradually as we go away from the surface. So, at the upper boundary, i.e., at the top of electrode layer we can have vanishing space charge density.

(I) Let us consider the case when there is no eddy diffusion. In this case, the eddy diffusion current term on the RHS of equation (1) is zero, i.e.,

$$
K(z) \frac{d \rho}{d z}=0 .
$$

Then in this case we have:

$$
I_{t}=E \lambda
$$


i.e., total current is carried only by conduction. From Poisson's equation we have:

$$
\rho=\varepsilon_{0} \nabla \cdot E .
$$

Taking divergence of electric field from (2) and substituting in (3) we get:

$$
\rho=\frac{\varepsilon_{0}}{\lambda} \nabla \cdot I_{t}-\frac{\varepsilon_{0}}{\lambda} E \cdot \nabla \lambda .
$$

Now, in the steady state conditions, $\nabla \cdot I_{t}=0$ and we get:

$$
\rho=-\frac{\varepsilon_{0}}{\lambda} E \cdot \nabla \lambda
$$

In one dimension, i.e., in vertical direction this equation becomes:

$$
\rho(z)=\frac{-\varepsilon_{0} E(z) \frac{d \lambda}{d z}}{\lambda(z)} .
$$

From equation (5) we can see that space charge density $\rho$ is directly proportional to the electric field and conductivity gradient and inversely proportional to the conductivity. Now here we consider two cases:

Case (A): $\lambda$ is constant with altitude

In this case $d \lambda / d z=0$ and from the above equation (6), $\rho=0$, which is by definition of electrode layer, not applicable to it.

Case (B): $\lambda$ is not constant with altitude

In this case since from equation (5) or (6) we have $\rho$ inversely proportional to $\lambda$, it will also change with altitude with change in $\lambda$. Also, from equation (2), $|E / \lambda|=\left|I_{t} / \lambda^{2}\right|$ or $=\left|E^{2} / I_{t}\right|$ is not linear. From the above definition of atmospheric electrode layer, with falling strength of electric field $E$ (fair weather atmospheric field is negative) as we go away from the surface (i.e., in other words with increasing conductivity $\lambda$ to fulfill equation 2), $|E / \lambda|$ is the largest at the surface. After differentiating equation (6) and using sign conventions as described above, we get nonzero $d \rho / d z$, i.e., $d \rho / d z \neq 0$ in electrode layer as follows:

Differentiating equation (6):

$$
\begin{aligned}
\frac{d \rho}{d z}= & -\varepsilon_{0} \frac{E(z)}{\lambda(z)} \frac{d^{2} \lambda}{d z^{2}}-\frac{\varepsilon_{0}}{\lambda(z)} \frac{d E}{d z} \frac{d \lambda}{d z} \\
& +\varepsilon_{0} \frac{E(z)}{\lambda(z)^{2}}\left(\frac{d \lambda}{d z}\right)^{2} .
\end{aligned}
$$

In the above equation (7) on RHS, considering fair weather electric field as negative, the last two terms are negative. Now, we should find out the sign of $d^{2} \lambda / d z^{2}$.

From the definition of electrode layer as given above, we should have nonzero $d \lambda / d z$ everywhere in electrode layer to have nonzero $\rho$ from equation (6). Moreover, it is positive from equation (6) since fair weather electric field is negative or vertical potential gradient is positive. Therefore, $d^{2} \lambda / d z^{2}$ exists. After rearranging terms, equation (6) can also be written as:

$$
1=\frac{-\varepsilon_{0} E(z)(d \lambda / d z)}{\lambda(z) \rho(z)} .
$$

From the above equation (8) we have larger value of $|E(z) / \lambda(z)|$ at the lower boundary and it gradually decreases as we go away from the surface when equation (2) is applicable. Therefore from equation (8), $[(d \lambda / d z) / \rho]$ should increase as we go away from the surface. This is achieved by the following three ways:

(i) $d \lambda / d z$ is constant and $\rho$ decreases,

(ii) $\rho$ is constant but $d \lambda / d z$ increases, and

(iii) both change.

For (i) and (iii) above, $\rho$ changes, so it proves the existence of $d \rho / d z$. For the case (ii) above, $\rho$ is constant and $d \lambda / d z$ increases. In reality since there is a negative sign in equation (8), it actually decreases as we go away from the surface and $d^{2} \lambda / d z^{2}$ will be negative for constant $\rho$ as mentioned in (ii) above, which contradicts equation (7) in which we get nonzero $d \rho / d z$ (i.e., $\rho$ is not constant) since all the terms on RHS are negative. So, we should have $\rho$ changing with altitude in electrode layer.

The above arguments lead to the conclusion that with the given definition of electrode layer and in horizontally homogeneous steady state conditions we have gradients of conductivity as well as space charge density at each level in electrode layer in the presence and absence of vertical eddy diffusion current. Further, the above logical arguments lead to the conclusion that $d \rho / d z$ is negative, i.e., $\rho$ decreases as we go away from the surface which is consistent with the previous model calculations in which $\rho$ becomes zero at the top of electrode layer.

Thus, we have proved that even in the absence of vertical eddy diffusion current, $d \rho / d z$ exists in electrode layer in steady state conditions.

(II) When the second term, i.e., vertical eddy diffusion current term on the RHS of current density equation (1) is nonzero, it automatically proves that $d \rho / d z \neq 0$. 
The above arguments lead to the conclusion that vertical space charge density gradient exists in electrode layer in the presence as well as in the absence of vertical eddy diffusion current in the steady state conditions.

From this we can conclude that in the steady state conditions and considering horizontal homogeneity, the quantity $[\rho /(d \rho / d z)]$ is well defined in electrode layer and we can derive the following.

2.2 Derivation of space charge density profile in electrode layer in the horizontally homogeneous and steady state conditions

As seen above, in electrode layer, $\rho$ and $d \rho / d z$ are nonzero, let us consider the following:

$$
\frac{\rho(z)}{(d \rho / d z)}=F(z)
$$

where $F(z)$ is any function of altitude $z$.

Since we are considering the condition of horizontal homogeneity, we consider Poisson's equation only in vertical direction as:

$$
\frac{d E}{d z}=\frac{\rho}{\varepsilon_{0}} .
$$

Now, from equation (9),

$$
\begin{gathered}
\frac{\rho}{\varepsilon_{0}}=F(z) \frac{1}{\varepsilon_{0}} \frac{d \rho}{d z}, \\
\text { i.e., } \frac{d E}{d z}=F(z) \frac{1}{\varepsilon_{0}} \frac{d \rho}{d z}, \\
\text { i.e., } \quad \int \frac{d E}{d z} d z=\frac{1}{\varepsilon_{0}} \int F(z) \frac{d \rho}{d z} d z,
\end{gathered}
$$

i.e., using integration by parts

$$
E=\frac{1}{\varepsilon_{0}} F(z) \rho-\int \frac{d F(z)}{d z} \rho(z) d z .
$$

Now the term in the integral sign is:

$$
F^{\prime}(z) \rho(z)=\frac{\rho(z) \rho^{\prime}(z)^{2}-\rho(z)^{2} \rho^{\prime \prime}(z)}{\rho^{\prime}(z)^{2}}
$$

where

$$
F^{\prime}(z)=\left.\frac{d F}{d z}\right|_{A t z}, \quad \rho^{\prime}(z)=\left.\frac{d \rho}{d z}\right|_{A t z}
$$

and

$$
\rho^{\prime \prime}(z)=\left.\frac{d^{2} \rho}{d z^{2}}\right|_{A t z}
$$

i.e., from equation (15) we get:

$$
\rho^{\prime}(z)^{2}\left(1-F^{\prime}(z)\right)=\rho(z) \rho^{\prime \prime}(z) .
$$

As mentioned above, in electrode layer we have $\rho$ and $\rho^{\prime}$ both decreasing with altitude when we go away from the surface, i.e., $\rho^{\prime}$ and $\rho^{\prime \prime}$ are negative. Further from the previous studies (e.g., Hoppel and Gathman 1971; Kulkarni and Kamra 2001) we can model electrode layer taking $\rho$ and $\rho^{\prime}$ to vanish at the same level, i.e., at the top of electrode layer. For achieving this upper boundary condition the percent rate of fall of $\rho$ and $\rho^{\prime}$ at the same altitude with respect to their surface value should be the same. This requires fulfillment of the following condition, i.e.,

$$
\frac{\rho(z)}{\rho^{\prime}(z)}=\frac{\rho^{\prime}(z)}{\rho^{\prime \prime}(z)}=F(z)
$$

or

$$
\rho^{\prime}(z)^{2}=\rho(z) \rho^{\prime \prime}(z)
$$

Therefore from equation (16) we should have $F^{\prime}(z)=0$, i.e., $F(z)$ is constant. We denote it by $c_{1}$.

In equation (14) the last integral on the RHS vanishes and finally we get:

$$
E(z)=c_{1} \frac{\rho(z)}{\varepsilon_{0}}+c_{2},
$$

where $c_{2}$ is the constant of integration:

$$
\Rightarrow \quad \frac{\rho}{(d \rho / d z)}=c_{1}=\text { constant } .
$$

From equation (20) we conclude that atmospheric space charge density has exponential variation with altitude and the profile is as follows:

$$
\int_{z_{0}}^{z} \frac{d \rho}{\rho} d z=\int_{z_{0}}^{z} \frac{d z}{c_{1}} .
$$


After integrating equation (21) we get profile of space charge density as:

$$
\rho(z)=\rho\left(z_{0}\right) e^{\left(z-z_{0} / c_{1}\right)} .
$$

From equation (22) we have an exponential variation of space charge density in electrode layer in the steady state conditions. Here, $z_{0}$ is chosen such that it should not be in the vicinity of the surface where molecular diffusion is predominant. We have derived the above profiles under the conditions of horizontal homogeneity and steady state. In atmospheric electricity, depending upon the relaxation time on the surface of the earth, hourly averages can be treated as the steady state values. For achieving horizontal homogeneity, there should not be tall objects such as hill, tree or tall building near the site of measurement (in about 1 to $2 \mathrm{~km}$ radius). Also, the site of measurement should be sufficiently plane and away from any local charge sources such as, high tension wires, industrial smoke, etc. Though theoretically defined electrode layer is disturbed by surface radioactivity, aerosol and turbulence over land, people have still observed presence of electrode layer space charge in the lowest meter on the surface of the earth (e.g., Law 1963; Crozier 1965). Knudsen et al (1989) have made measurements in electrode layer over snow surface. All the above points show that it is possible to make measurements in electrode layer in the lowest meter. So, the lower boundary $z_{0}$ should be in the lowest meter on the surface.

From the basic knowledge of boundary layer, it is quite reasonable to assume the linear profile of eddy diffusivity in surface layer. If we allow $K(z)=K z$ (Willett 1978) and use equation (20) for space charge density gradient and equation (19) for electric field in current density equation (1), we get:

$$
I_{t}=\left(\frac{c_{1} \rho(z)}{\varepsilon_{0}}+c_{2}\right) \lambda(z)-K(z) \frac{\rho(z)}{c_{1}} .
$$

From equation (23) above, we have profile of conductivity as:

$$
\lambda(z)=\frac{I_{t}+A z \rho(z)}{B \rho(z)+c_{2}},
$$

where $A=K / c_{1}$ and $B=c_{1} / \varepsilon_{0}$ and $\rho(z)$ is given by equation (22).

After rearranging the terms in equation (23), we get:

$$
K(z) \frac{\rho(z)}{c_{1}}=\frac{c_{1} \rho(z) \lambda(z)}{\varepsilon_{0}}+c_{2} \lambda(z)-I_{t}
$$

The profile of eddy diffusion coefficient used by Willett (1978) can be used which is of the type $K(z)=k u^{*} z$ where $k$ is a constant $=0.4$ and $u^{*}$ is friction velocity, $k u^{*}=K$, is a constant in $\mathrm{m} \mathrm{s}^{-1}$ and $z$ is an altitude in meters.

$$
\therefore \quad K(z)=K z=\frac{c_{1}^{2} \lambda(z)}{\varepsilon_{0}}+\frac{c_{1} c_{2} \lambda(z)}{\rho(z)}-\frac{I_{t} c_{1}}{\rho(z)} .
$$

We have three measured quantities, electrical conductivity $\lambda$, total current density $I_{t}$, space charge density $\rho$ and three unknowns namely, $K$, $c_{1}$ and $c_{2}$. Therefore, we require three equations. Assuming linear profile of eddy diffusion coefficient in the surface layer as given above, for known altitudes $z_{1}, z_{2}$ and $z_{3}$, we have:

$$
\begin{aligned}
& K\left(z_{1}\right)=K z_{1}=\frac{c_{1}^{2} \lambda_{1}}{\varepsilon_{0}}+\frac{c_{1} c_{2} \lambda_{1}}{\rho_{1}}-\frac{I_{t} c_{1}}{\rho_{1}}, \\
& K\left(z_{2}\right)=K z_{2}=\frac{c_{1}^{2} \lambda_{2}}{\varepsilon_{0}}+\frac{c_{1} c_{2} \lambda_{2}}{\rho_{2}}-\frac{I_{t} c_{1}}{\rho_{2}}, \\
& K\left(z_{3}\right)=K z_{3}=\frac{c_{1}^{2} \lambda_{3}}{\varepsilon_{0}}+\frac{c_{1} c_{2} \lambda_{3}}{\rho_{3}}-\frac{I_{t} c_{1}}{\rho_{3}},
\end{aligned}
$$

where $\lambda_{1}, \lambda_{2}, \lambda_{3}$ and $\rho_{1}, \rho_{2}$ and $\rho_{3}$ are the measured conductivities and space charge densities at heights $z_{1}, z_{2}$ and $z_{3}$, respectively. These three equations can be solved to get three unknowns $c_{1}, c_{2}$ and $K$. Israelsson et al (1993) give procedure for measuring conductivity at different altitudes. Similar is the procedure for space charge density measurement. Also, Kamra (1982), Knudsen et al (1989), Pawar and Kamra (2000) measure space charge density at different altitudes near the surface of the earth.

In spite of this, these three levels of measurements can further be reduced to two and then to only one by using the following:

$c_{2}$ can be used in terms of $c_{1}$ from equation (19) and making measurement of $E$ at the lower level $z_{0}$ and using profile of space charge density one can get:

$$
\begin{aligned}
K(z)= & K z=\frac{c_{1}^{2} \lambda(z)}{\varepsilon_{0}}+\frac{c_{1} \lambda(z)\left(E\left(z_{0}\right)-\left(c_{1} / \varepsilon_{0}\right) \rho\left(z_{0}\right)\right)}{\rho\left(z_{0}\right) e^{\left(z-z_{0}\right) / c_{1}}} \\
& -\frac{I_{t} c_{1}}{\rho\left(z_{0}\right) e^{\left(z-z_{0}\right) / c_{1}}} .
\end{aligned}
$$

If equation (30) is used it is required to measure either (i) electric field and space charge density at $z_{0}$, air earth current density by plate antenna which 
is kept flush with the surface and conductivity at suitable two altitudes or (ii) space charge density at $z_{0}$ and $z, E\left(z_{0}\right), I_{t}$ and $\lambda(z)$ to find out $K, c_{1}$ and $c_{2}$, i.e., we require two levels of measurement $z_{0}$ and $z$ either for space charge density or for conductivity. These measurements are quite easy to make (e.g., Kamra 1982; Knudsen et al 1989; Israelsson et al 1993; Pawar and Kamra 2000).

Now, equation (26) can further be reduced into only one unknown and needs only one level of measurement $z_{0}$ as given below:

$$
\begin{aligned}
I_{t} & =E\left(z_{0}\right) \lambda\left(z_{0}\right)-K z_{0} \frac{\rho\left(z_{0}\right)}{c_{1}} \\
\frac{K}{c_{1}} & =\frac{E\left(z_{0}\right) \lambda\left(z_{0}\right)-I_{t}}{\rho\left(z_{0}\right) z_{0}} .
\end{aligned}
$$

In the above expression, from the measurements of $E, \lambda$ and $\rho$ at $z_{0}$ and of $I_{t}$ with the plate antenna kept flush with the ground we get $K / c_{1}$.

Though theoretically we can model space charge density to be zero at the top of electrode layer, practically it means that the amount of charge density which does not give rise to appreciable $d E / d z$ and electric field can be treated as almost constant with respect to altitude. From Poisson's equation we can see that when space charge density becomes two orders of magnitude smaller than $\varepsilon_{0}$ we can get almost constant electric field with altitude, i.e., $d E / d z \rightarrow 0$. Now we know the profile of space charge density from equation (22). We measure $\rho$ at the lower boundary $z_{0}$. We can find out ratio $\left(z-z_{0}\right) / c_{1}$ for some $z$ for which $\rho(z)$ becomes negligible and does not have any appreciable effect on the gradient of electric field. This is nothing but the ratio $L_{t} / c_{1}$, where $L_{t}$ is the thickness of electrode layer. Let this ratio be equal to $R$, i.e., we are finding the height at which we have:

$$
\begin{gathered}
\rho(z) \approx 10^{-14}=\rho\left(z_{0}\right) e^{\left(L_{t} / c_{1}\right)} \\
\varepsilon_{0}=8.85 \times 10^{-12} \mathrm{~F} \mathrm{~m}^{-1} .
\end{gathered}
$$

Now, we know $K / c_{1}$ (equation 32) as well as $R=L_{t} / c_{1}$ (equation 33).

From the expression of $L_{t}$ and the above expression (32) for $K / c_{1}$ we get:

$$
\frac{L_{t}}{c_{1}}=R=\frac{2 \varepsilon_{0}}{\lambda(\infty)}\left(\frac{E(0) \lambda(0)-I_{t}}{\rho\left(z_{0}\right) z_{0}}\right)
$$

From the above expression we can get the value of $\lambda(\infty)$. Also from equation (19) we get:

$$
c_{2}=\left(E\left(z_{0}\right)-\frac{c_{1}}{\varepsilon_{0}} \rho\left(z_{0}\right)\right) .
$$

Further, at the top of electrode layer, since $I_{t}=$ $E(\infty) \cdot \lambda(\infty), c_{2}=E(\infty)$ can be found from the measured $I_{t}$. From the expression of electric field, we can have $c_{2}$ in terms of $c_{1}$ as given in equation (35). So, we can also find out $c_{1}$. Finally, as we know $K / c_{1}$ and $c_{1}, K$ can be found out.

Looking at equations (31)-(34) we see that just making measurements at lower boundary $z_{0}$ we can get all $c_{1}, c_{2}$ and $K$. So, from the above equations we see that we can solve single equation for single unknown at $z_{0}$ near the surface by making measurements of $\rho, \lambda$ and $E$ at $z_{0}$. $I_{t}$ can be measured by plate antenna which is kept flush with the ground. All this measurement system is well established in atmospheric electricity. All characteristic parameters or scales of an electrode layer such as $E(\infty)$, $\lambda(\infty), L_{t}$ and $K$ can be found from only surface measurements. There is no need to know ionization rate $q$ and total aerosol concentration $Z$.

Since scientists have used atmospheric electrical approach for determining eddy diffusion coefficient, the above method can be used for comparison as well as for determining other scales of an electrode layer. The procedure is given below. This method does not involve any major assumption and so can be used in fair weather conditions for electrode layer studies.

Hoppel and Gathman (1971), Willett (1978, 1983), Kulkarni and Kamra (2001) show that the equations governing the flux of polar ion densities are non-linear hyperbolic. Therefore, the solutions which are attained after using proper lower and upper boundary conditions are asymptotic. These final asymptotic values of electric field, conductivity and individual ion and aerosol densities define the electrical structure of electrode layer. Under the steady state conditions and assuming ions are formed in pairs, these final polar ion densities are equal in magnitude. Similar is the case with charged aerosol particles also. Therefore when these steady state values are reached, total space charge density approaches zero. So, theoretically, from equation (19) we have:

when $E=E(\infty), \rho=0$, where $E(\infty)$ is the steady state asymptotic value of electric field.

$$
\therefore \quad E(\infty)=c_{2} .
$$


Table 1. Calculated $c_{1}$ for the measurements by Law (1963).

\begin{tabular}{|c|c|c|c|c|c|c|}
\hline $\begin{array}{l}I_{t} \\
\left(\text { ele } \mathrm{cm}^{-2} \mathrm{~s}^{-1}\right)\end{array}$ & $\begin{array}{c}z \\
(\mathrm{~m})\end{array}$ & $\begin{array}{c}E \\
\left(\mathrm{~V} \mathrm{~m}^{-1}\right)\end{array}$ & $\begin{array}{c}\lambda \\
\mathrm{fS} \mathrm{m}^{-1}\end{array}$ & $\begin{array}{c}\rho \\
(\mu \mu \mathrm{c})\end{array}$ & $\begin{array}{c}K \\
\left(\mathrm{~m}^{2} \mathrm{~s}^{-1}\right)\end{array}$ & $\begin{array}{c}c_{1} \\
(\mathrm{~m})\end{array}$ \\
\hline \multicolumn{7}{|c|}{ Day measurements } \\
\hline 80 & 1 & -72 & 5.9 & 7.2 & 0.1 & -2.4 \\
\hline 80 & 1.5 & -71.5 & 6.1 & 7.0 & 0.1 & -2.3 \\
\hline \multicolumn{7}{|c|}{ Night measurements } \\
\hline 110 & 1 & -17.5 & 5.9 & -18.4 & 0.01 & 2.5 \\
\hline 110 & 1.5 & -18.5 & 6.4 & -15.0 & 0.01 & 2.6 \\
\hline
\end{tabular}

$c_{1}$ has dimension of length; total current $\left(I_{t}\right)$ is given in CGS units.

Table 2. Measurements of Kamra (1982) and calculated $c_{1}$.

\begin{tabular}{lcccccc}
\hline & Daytime & & & \multicolumn{3}{c}{ Night-time } \\
\cline { 1 - 1 } $\begin{array}{c}\text { Local time } \\
(\mathrm{h})\end{array}$ & $\begin{array}{c}\rho \text { at } 0.1 \mathrm{~m} \\
(\mu \mu \mathrm{c})\end{array}$ & $\begin{array}{c}\rho \text { at } 1 \mathrm{~m} \\
(\mu \mu \mathrm{c})\end{array}$ & & $\begin{array}{c}\text { Local time } \\
(\mathrm{h})\end{array}$ & $\begin{array}{c}\rho \text { at } 0.1 \mathrm{~m} \\
(\mu \mu \mathrm{c})\end{array}$ & $\begin{array}{c}\rho \text { at } 1 \mathrm{~m} \\
(\mu \mu \mathrm{c})\end{array}$ \\
\hline $0800-1200$ & 5 & 60 & & $2000-2400$ & -450 & -400 \\
$1200-1600$ & 38 & 20 & & $0000-0004$ & -900 & -800 \\
$1600-2000$ & 36 & Small & & $0004-0008$ & -700 & -800 \\
Average & 26.3 & 26.7 & & Average & -683.3 & -666.7 \\
Calculated $c_{1}$ & -3.7 & -3.8 & & Calculated $c_{1}$ & 10.0 & 9.8 \\
\hline
\end{tabular}

Substituting this value of $c_{2}$ in equation (19) one can write:

$$
\begin{aligned}
E(z) & =\frac{c_{1} \rho(z)}{\varepsilon_{0}}+E(\infty), \\
\therefore \quad c_{1} & =\frac{(E(z)-E(\infty)) \varepsilon_{0}}{\rho(z)}, \\
\left(\frac{d \rho}{d z}\right)_{z} & =\frac{\rho(z)}{c_{1}}=\frac{\rho(z)}{\left(E(z)-E(\infty) \varepsilon_{0}\right) / \rho(z)} \\
& =\frac{\rho(z)^{2}}{(E(z)-E(\infty)) \varepsilon_{0}} .
\end{aligned}
$$

Also, when the steady state conditions are reached we have total current density $I_{t}=E(\infty) \lambda(\infty)$ where $\lambda(\infty)$ is the steady state conductivity.

Looking at the above equations we see that in electrode layer since $c_{2}$ is the steady state asymptotic electric field, it is constant. Also, in the profile of eddy diffusion coefficient $K(z)=K z, K$ is constant. So, in the above equations it is only required to confirm from various datasets whether $c_{1}$ is nearly constant in case of actual measurements and in previous model results. In the next part we discuss some of the previous measurements and theoretical results to confirm the utility of this
Table 3. Results of Willett (1983) and our calculated $c_{1}$.

\begin{tabular}{lccc}
\hline$z_{i}$ & \multicolumn{1}{c}{$J_{i}$} & $\rho_{i}$ & $c_{1}$ \\
\hline \multicolumn{2}{l}{ Aerosol-free case } \\
$10^{-5}$ & -0.333 & 3.2 & $-9.8 \times 10^{-6}$ \\
$10^{-4}$ & -0.036 & 3.6 & $-10.2 \times 10^{-6}$ \\
\multicolumn{2}{l}{ Aerosol-dominated case } & & \\
$10^{-1}$ & -0.70 & 3.6 & -0.04 \\
$10^{-2}$ & -0.14 & 4.9 & -0.03 \\
\hline
\end{tabular}

Eddy diffusion coefficient $K=k u^{*} z i$ where $k$ is constant $=0.4$ and friction velocity $u^{*}=0.256 \mathrm{~m} \mathrm{~s}^{-1}$ for aerosol-free case and $0.2 \mathrm{~m} \mathrm{~s}^{-1}$ for aerosol-dominated case. Total current density $I_{t}=-2.7 \mathrm{pA} \mathrm{m}^{-2}$ for aerosolfree atmosphere and $-1.3 \mathrm{pA} \mathrm{m}^{-2}$ for aerosol-dominated case.

method and practical validity of derived equations. For convenience, calculations are done using two altitudes near the surface of the earth. We use equations (36)-(39) for this comparison.

\section{Comparison with previous theoretical results and measurements}

First we consider the results of Law (1963) based on measurements. Table 1 displays Law's data for different parameters along with our calculated $c_{1}$ values at two different altitudes.

Table 2 displays measurements of Kamra (1982) and our calculated $c_{1}$ values. These values in table 2 
Table 4. Model generated values (Hoppel and Gathman 1971) of electric field and space charge density at different levels for surface electric field $E_{0}=-100 \mathrm{~V} \mathrm{~m}^{-1}$.

\begin{tabular}{lccccc}
\hline $\begin{array}{l}\text { Mixing parameter } \\
\chi\left(\mathrm{m}^{2} \mathrm{~s}^{-1}\right)\end{array}$ & $\begin{array}{c}-E \text { at } 10 \mathrm{~m} \\
\mathrm{~V} \mathrm{~m}^{-1}\end{array}$ & $\begin{array}{c}-E \text { at } 30 \mathrm{~m} \\
\mathrm{~V} \mathrm{~m}{ }^{-1}\end{array}$ & $\begin{array}{c}\rho \text { at } 1 \mathrm{~m} \\
\times 10^{6} \mathrm{ele} \cdot \mathrm{m}^{-3}\end{array}$ & $\begin{array}{c}\rho \text { at } 10 \mathrm{~m} \\
\times 10^{6} \mathrm{ele} \cdot \mathrm{m}^{-3}\end{array}$ & $\begin{array}{c}\rho \text { at } 30 \mathrm{~m} \\
\times 10^{6} \mathrm{ele} \cdot \mathrm{m}^{-3}\end{array}$ \\
\hline $\begin{array}{l}\text { Nucleus free atmosphere } \\
12\end{array}$ & 78.6 & 63.3 & 185 & 70 & 26 \\
6 & 70.6 & 55.5 & 283 & 79 & 20 \\
3 & 62.3 & 50 & 401 & 82 & - \\
500 Nuclei per cc & & & & 63 & - \\
12 & 81.6 & 66.7 & 152 & 72 & - \\
6 & 74.7 & 61.4 & 234 & & \\
\hline
\end{tabular}

Table 5. Calculated values of $c_{1}$ and conductivity using data in table 4.

\begin{tabular}{lcccc}
\hline & $\begin{array}{c}\text { Calculated } c_{1} \\
\text { at } 10 \mathrm{~m}\end{array}$ & $\begin{array}{c}\text { Calculated } \lambda \\
\left.(\mathrm{fS} \mathrm{m})^{-1}\right) \\
\text { at } 10 \mathrm{~m}\end{array}$ & $\begin{array}{c}\text { Calculated } \lambda \\
\left.(\mathrm{fS} \mathrm{m})^{-1}\right) \\
\text { at } 30 \mathrm{~m}\end{array}$ & $\begin{array}{c}\text { Calculated } \lambda^{\prime} \\
\left(\mathrm{fS} \mathrm{m}^{-1}\right) \\
\text { using } c_{1} \text { at } 30 \mathrm{~m}\end{array}$ \\
\hline Clean atmosphere & & & & 34.5 \\
12 & -24.0 & 28.1 & 32.5 & 35.8 \\
6 & -15.7 & 30.3 & 34.6 & - \\
3 & -09.6 & 33.3 & - & - \\
500 Nuclei per cc & & & - & - \\
12 & -26.0 & 19.9 & - & \\
6 & -20.51 & 20.8 & & \\
\hline
\end{tabular}

indicate daytime and night-time averages. For the daytime, average convection current density in the first meter is $-0.71 \mathrm{pA} \mathrm{m}^{-2}$ and $K=0.1 \mathrm{~m}^{2} \mathrm{~s}^{-1}$ and that for the night-time is $-0.68 \mathrm{pA} \mathrm{m}^{-2}$ and $K=0.01 \mathrm{~m}^{2} \mathrm{~s}^{-1}$.

Willett (1983) has obtained theoretical results for dimensionless parameters under different conditions. Table 3 indicates his results for two cases namely aerosol-free and aerosol-dominated atmosphere. $z_{i}, J_{i}$ and $\rho_{i}$ are the dimensionless height, convection current density and space charge density respectively.

From the above three tables we see that $c_{1}$ at two different altitudes is nearly equal near the surface of the earth. So it is suggested that in the study of electrode layer one can use theoretically derived exponential profile of space charge density. Further, from the given equations we can get different characteristic parameters of electrode layer as, eddy diffusion coefficient $K, c_{2}$, i.e., steady state electric field $E(\infty)$, steady state conductivity $\lambda(\infty)\left(=\right.$ total current $\left.I_{t} / c_{2}\right)$ and from the derived profile of space charge density, we get the thickness of electrode layer. Also, we get profiles of electric field and conductivity. From $\lambda(\infty)$ one can get the average aerosol concentration as well.

At the end, we see solutions by Hoppel and Gathman (1971). Table 4 displays model generated results of Hoppel and Gathman (1971).
Total current density for the clean atmosphere is $-1.7 \mathrm{pA} \mathrm{m}^{-2}$ and that for 500 nuclei content per $\mathrm{cm}^{-3}$ is $-1.2 \mathrm{pA} \mathrm{m}^{-2}$. Asymptotic electric field $E(\infty)$ is $-48 \mathrm{~V} \mathrm{~m}^{-1}$. Table 5 displays our calculated $c_{1}$ at $10 \mathrm{~m}$ and conductivities for the data in table 4 . The conductivity at $30 \mathrm{~m}$ is calculated using two procedures: (i) by model generated values incorporating into the current density equation and (ii) by using $c_{1}$ calculated at $10 \mathrm{~m}$. So, with this, it is checked that whether $c_{1}$ calculated at one level $(10 \mathrm{~m})$ gives resulting value of $\lambda$ at other level $(30 \mathrm{~m})$ which agrees well with their model results. Since these results are numerical solutions of hyperbolic equations, we can not expect $c_{1}$ at some level to be exactly equal to that at other level. In spite of this, we can still judge the agreement of calculated conductivities with their model results. As altitude increases, solutions tend to become asymptotic and by definition of electrode layer, it is a region of space charge. So we use $c_{1}$ calculated at lower level. $c_{1}$ is calculated at $10 \mathrm{~m}$. Further, Hoppel and Gathman (1971) have also made measurements over open-ocean. Here data of May 6, 1969 are used for convenience since the conditions at that time are nearly similar to the conditions they have used in model. Table 6 displays measurements taken by them, their numerically calculated $\chi$ and $\lambda$ and our calculated $\lambda$. Next, we use their results for calculating thickness of an electrode layer for different 
Table 6. Comparison of conductivities at $1.5 \mathrm{~m}$ from model solution of Hoppel and Gathman (1971) for mixing parameter $\chi=12 \mathrm{~m}^{2} \mathrm{~s}^{-1}$ and nuclei 500 per $c c$ and calculated using the procedure outlined above for their actual measurements.

\begin{tabular}{|c|c|c|c|c|c|}
\hline $\begin{array}{c}-E \text { at } 1.5 \mathrm{~m} \\
\left(\mathrm{~V} \mathrm{~m}^{-1}\right) \\
\text { (measured) }\end{array}$ & $\begin{array}{c}\rho \text { at } 1.5 \mathrm{~m} \\
\times 10^{6}\left(\mathrm{ele} \cdot \mathrm{m}^{-3}\right) \\
(\text { measured })\end{array}$ & $\begin{array}{c}\mathrm{Z} \\
\times 10^{6}\left(\mathrm{~m}^{-3}\right) \\
\text { (measured) }\end{array}$ & $\begin{array}{c}{\left(\mathrm{m}^{2} \mathrm{~s}^{-1}\right)}_{\text {determined }} \\
\text { from model }\end{array}$ & $\begin{array}{c}\lambda \\
\left(\mathrm{fS} \mathrm{m}^{-1}\right) \\
\text { determined } \\
\text { from model }\end{array}$ & $\begin{array}{c}\lambda \\
\left(\text { fS } \mathrm{m}^{-1}\right) \\
\text { calculated } \\
\text { by our method }\end{array}$ \\
\hline 101 & 161 & 501 & $10 \pm 6$ & $\sim 15.4$ & $\sim 14.5$ \\
\hline
\end{tabular}

Table 7. Different mixing strengths and the corresponding thickness of electrode layer for the data in table 4 (Hoppel and Gathman 1971) for aerosol-free atmosphere.

\begin{tabular}{cc}
$\begin{array}{c}\text { Mixing parameter } \\
\chi\left(\mathrm{m}^{2} \mathrm{~s}^{-1}\right)\end{array}$ & $\begin{array}{c}\text { Electrode layer } \\
\text { thickness } L_{t}(\mathrm{~m})\end{array}$ \\
\hline 3 & 38.65 \\
6 & 54.66 \\
12 & 77.29 \\
\hline
\end{tabular}

mixing strengths. Table 7 displays thickness of an electrode layer for the data in table 4 . We calculate thickness $L_{t}$ using the following standard expression (Kulkarni and Kamra 2001):

$$
L_{t}=\left(\frac{2 \varepsilon_{0} \chi}{\lambda(\infty)}\right)^{1 / 2}
$$

Figure 1 shows space charge density profiles calculated from equation (22) starting from $1 \mathrm{~m}$ for different mixing strengths. Initial values for $\rho$ at $1 \mathrm{~m}$ are taken from table 4.

As seen from table 5, calculated values of conductivity at $30 \mathrm{~m}$ are in reasonable agreement with each other. Similar is the case with table 6 also. If we compare figure 1 with the values given in table 7 for electrode layer thickness, they are in very good agreement. (Calculated quantities can also be confirmed with the graphical solutions given by Hoppel and Gathman 1971.)

As the thickness of electrode layer calculated by traditional formula and that using the profile of space charge density derived here are in very good agreement, it indicates that the other two characteristic scale parameters $K$ and $\lambda(\infty)$ are also determined with sufficient accuracy. Consequently, steady state electric field $E(\infty)$, i.e., $c_{2}$ is also determined with sufficient accuracy for the measured $I_{t}$. In most of the cases we see that constant $c_{1}$ is nearly the same at two different levels for that particular dataset which proves the validity of exponential variation of space charge density. So we can call $c_{1}$ as electrode layer constant. It has dimension of length.

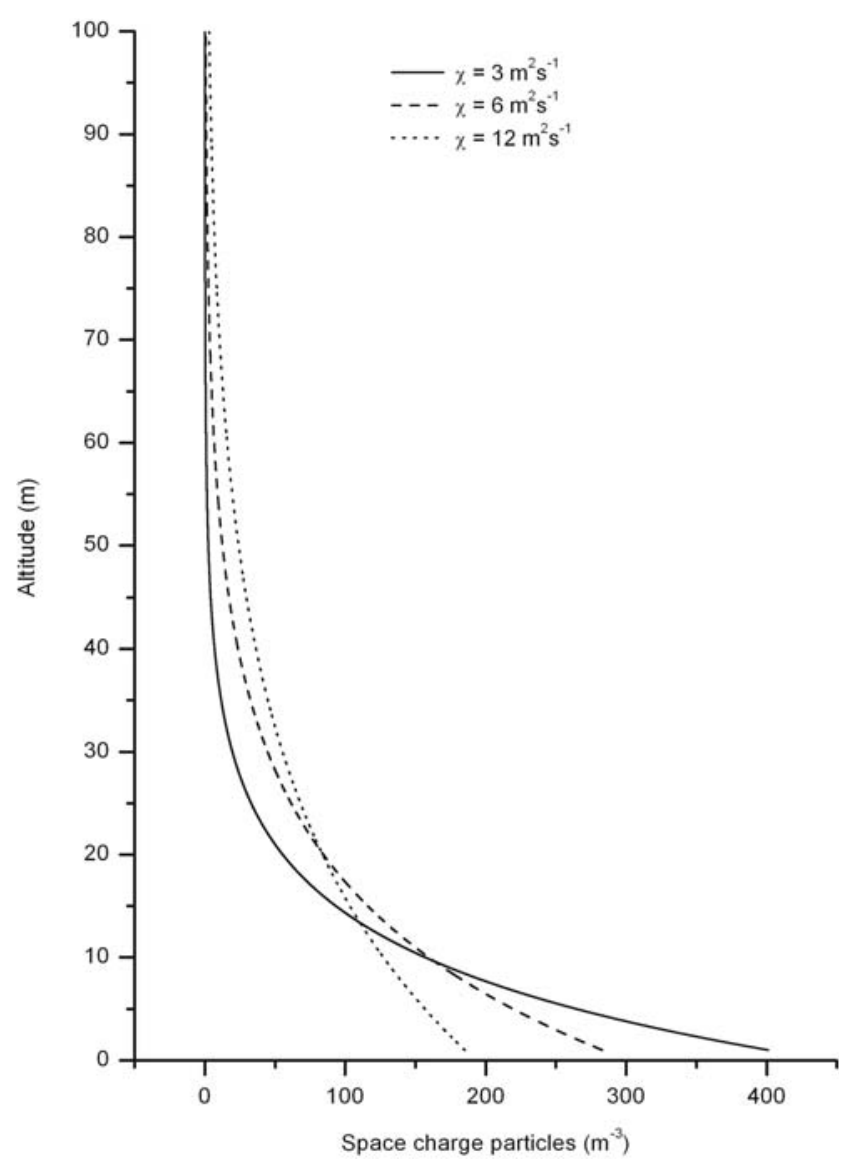

Figure 1. Space charge density profiles calculated by equation (22) to show electrode layer thickness $L_{t}$ for different mixing strengths.

If we use average value of conductivity $\left(\lambda_{a}\right)$ and eddy diffusion coefficient $\left(K_{a}\right)$ in the total current density equation (1), after differentiating we get second order linear differential equation as follows:

$$
\begin{aligned}
& K_{a} \frac{d^{2} \rho(z)}{d z^{2}}-\frac{\lambda_{a} \rho(z)}{\varepsilon_{0}}=0, \quad \because \frac{d I_{t}}{d z}=0, \\
& \frac{d \lambda_{a}}{d z}=0 \quad \text { and } \quad \frac{d K_{a}}{d z}=0 .
\end{aligned}
$$

After solving the above linear differential equation (41) for $\rho$ we get again an exponential 
profile of space charge density $\rho$ as:

$$
\rho(z)=A_{1} e^{z \sqrt{\lambda_{a} /\left(\varepsilon_{0} K_{a}\right)}}+A_{2} e^{-z \sqrt{\lambda_{a} /\left(\varepsilon_{0} K_{a}\right)}},
$$

i.e., of the type

$$
\rho(z)=A_{1} e^{\left(-z / c_{1}\right)}+A_{2} e^{\left(z / c_{1}\right)}
$$

where

$$
c_{1}=-\sqrt{\frac{\varepsilon_{0} K_{a}}{\lambda_{a}}}
$$

$A_{1}$ and $A_{2}$ can be found from boundary conditions. We know that as $z \rightarrow \infty$ (theoretically), $\rho \rightarrow 0$. So, in equation (42), $A_{1}$ should equal zero. Further, at $z=z_{0}, \rho(z)=\rho\left(z_{0}\right)$ where $z_{0}$ is the lower boundary near the surface. Therefore, equation (42) becomes,

$$
\rho(z)=\rho\left(z_{0}\right) e^{\left(z-z_{0}\right) / c_{1}},
$$

which is same as equation (22), where $c_{1}=$ $-\left(\varepsilon_{0} K_{a} / \lambda_{a}\right)^{1 / 2}$, i.e., $c_{1}^{2}=\varepsilon_{0} K_{a} / \lambda_{a}, K_{a}$ can be calculated from the definition of average and using profile of eddy diffusion coefficient $K(z)$ as $K(z)=$ $K z$ (Willett 1978, 1983):

$$
K_{a}=\frac{\int_{z_{0}}^{z} K z d z}{\int_{z_{0}}^{z} d z}=\frac{K}{2}\left(z+z_{0}\right) .
$$

As expected from the expression of $c_{1}$ given above in terms of average values of conductivity and eddy diffusivity, in table 5 we see that $c_{1}$ increases with increase in turbulence strength and with the presence of aerosols. Since $K_{a}$ and $\lambda_{a}$ define average meteorological and electrical state of the atmosphere one can expect good correlation of these parameters with ambient environmental or meteorological parameters.

The major advantage of this method is that it does not involve any major assumption. It involves surface measurements which one can make with proper care. It gives information about all scales of an electrode layer and profiles of space charge density, electric field and conductivity above molecular diffusion layer with sufficient accuracy. It is expected that study of diurnal and seasonal variations in these will be useful to get more correlations with respect to different meteorological phenomena and in understanding processes occurring near the surface.
Major limitation while using this method is about the choice of the site of measurement. Also, one should confirm the existence of atmospheric electrode effect in the measurements and the corresponding time scale before drawing any conclusion. This is discussed in more detail by Tuomi (1980).

\section{Conclusions}

Though electrode layer is one of the charge generators in the global atmospheric electric circuit, in the literature, we find few studies on it. A new system of basic equations is used here. Using a new technique, it is shown that the derived profile of space charge density in electrode layer is exponential in the fair weather conditions under the conditions of steady state and horizontal homogeneity. Also, a new experimental method based on the surface measurements is discussed to get information about different characteristic parameters of an electrode layer, profiles of electric field and conductivity and also an average electrical and meteorological state of an environment. Further, use of this theoretical formulation with the previous model results and measurements confirms its utility in the fair weather atmospheric conditions. So, it is suggested that one can use theoretically derived exponential charge density profile for studying electrode layer with sufficient accuracy.

\section{Acknowledgements}

I gratefully acknowledge the support, infrastructure and opportunity given by Prof. B N Goswami, Director, Indian Institute of Tropical Meteorology, Pune, India, Dr A K Kamra, Former Head of Instrumentation Division and Dr S D Pawar, Head of Instrumentation Division. Also, my thanks are due to Mr V Gopalakrishnan and Mr P Murugavel for their technical help.

\section{References}

Clark J F 1958 Fair weather atmospheric electric potential and its gradient; In: Recent advances in atmospheric electricity (ed.) Smith L G, p. 67.

Crozier W D 1965 Atmospheric electrical profiles below three meters; J. Geophys. Res. 70 2785-2792.

Dolezalek H 1978 WMO Tech. Note No. 162, p. 60.

Hoppel W A 1967 Theory of the electrode effect; J. Atmos. Terr. Phys. 29 709-721.

Hoppel W A and Gathman S G 1971 Determination of eddy diffusion coefficient from atmospheric electrical measurements; J. Geophys. Res. 76 1467-1477.

Israel H 1973 Atmospheric electricity, Vol. II, IPST, Jerusalem. 
Israelsson S, Knudsen E and Anisimov S V 1993 Vertical profiles of electrical conductivity in the lowermost part of the turbulent boundary layer over flat ground; J. Atmos. Terr. Phys. 56 1545-1550.

Kamra A K 1982 Fair weather space charge distribution in the lowest two meters of the atmosphere; J. Geophys. Res. 87 4257-4263.

Kawano M 1957 The coefficient of eddy diffusivity estimated by the method of atmospheric electricity; J. Meteor. Soc. Japan 35 339-342.

Knudsen E, Israelsson S and Hallberg B 1989 Measurement of the electrode effect over flat snow-covered ground; J. Atmos. Terr. Phys. 51 521-527.

Kraakevik J H 1958 Electrical conduction and convection currents in the troposphere; In: Recent advances in atmospheric electricity (ed.) Smith L G, p. 81.

Kulkarni M and Kamra A K 2001 Vertical profiles of atmospheric electric parameters close to ground; J. Geophys. Res. 106 28,209-28,221.

Law J 1963 The ionization of the atmosphere near the ground in fair weather; Quart J. Roy. Met. Soc. 89 107-121.

Markson R and Sedlacek J 1981 Turbulent transport of electric charge in the marine amospheric boundary layer; J. Geophys. Res. 86 12,115-12,121.
Pawar S D and Kamra A K 2000 Comparative measurements of the atmospheric electric space charge density made with the filtration and Faraday cage technique; Atmos. Res. 54 105-116.

Raina P N 1984 On the validity of Ohm's law in the atmospheric electric circuit, Ph.D. Thesis, University of Kashmir, India.

Tammet H, Israelsson S, Knudsen E and Tuomi T 1996 Effective area of a horizontal long-wire antenna collecting the amospheric electric vertical current; J. Geophys. Res. $10129,672-29,678$.

Tuomi T J 1980 Atmospheric electrode effect: Approximate theory and wintertime observations; Pure Appl. Geophys. $11931-37$.

Varshneya N C 1983 Role of space charge in global atmospheric electricity: A Mathematical model; In: Proceedings in Atmospheric Electricity (eds) Ruhnke L H and Latham J, p. 94.

Willett J C 1978 An analysis of the electrode effect in the limit of strong turbulent mixing; J. Geophys. Res. 83 402-408.

Willett J C 1983 The turbulent electrode effect as influenced by interfacial ion transfer; J. Geophys. Res. 88 8453-8469. 